PERSPECTIVE

\title{
Electronic Health Records and Transgender Patients-Practical Recommendations for the Collection of Gender Identity Data
}

\author{
Madeline B. Deutsch, M.D. ${ }^{7}$ and David Buchholz, M.D. ${ }^{2}$ \\ 'Department of Family \& Community Medicine, University of California, San Francisco, CA, USA; ${ }^{2}$ Department of Pediatrics, University of California, \\ San Francisco, CA, USA.
}

Transgender (Trans, Trans*) persons may have a gender identity and a preferred name that differ from those assigned at birth, and/or those listed on their current legal identification (Gender ID, Birth-assigned Sex, Legal Sex). Transgender people who are referred to in a clinical setting using the wrong pronoun or name may suffer distress, ridicule or even assault by others in the waiting area, and may not return for further care. Furthermore, failure to accurately document (and therefore count) transgender identities has negative implications on quality improvement and research efforts, funding priorities and policy activities. The recent announcement that gender identity data may be included in Meaningful Use Stage 3 has accelerated the need for guidance for both vendors and local implementation teams on how to best record and store these data. A recent study demonstrated wide variation in current practices. This manuscript provides a description of identifiers associated with gender identity, and makes practical and evidence based recommendations for implementation and front-end functionality.

KEY WORDS: electronic health records; transgender; preferred name; preferred pronoun; legal sex; gender identity.

J Gen Intern Med 30(6):843-847

DOI: $10.1007 / \mathrm{s} 11606-014-3148-7$

(C) Society of General Internal Medicine 2014

\section{INTRODUCTION}

Transgender (Trans, Trans*) persons may have a gender identity and a preferred name that differ from those assigned at birth, and/or those listed on their current legal identification (Gender Identification, Birth-assigned Sex, Legal Sex). Recent estimates indicate that as many as $0.5 \%$ of the US population identifies as transgender, a population roughly equal to the US population of Hawaiian and Pacific Islanders. ${ }^{1,2}$ Transgender people who are referred to in a clinical setting using the wrong pronoun or name may suffer distress, ridicule or even assault by others in the waiting area, and may not return for further care. Transgender people face intense health disparities and lack of access to care; failure to accurately document (and therefore count) transgender identities furthers these disparities through negative implications on

Received July 18, 2014

Revised October 25, 2014

Accepted December 5, 2014

Published online January 6, 2015 quality improvement and research efforts, funding priorities and policy activities. ${ }^{3,4,5}$

"Gender ID data" can be defined as gender ID, birthassigned sex, legal sex, preferred name, and legal name. The US General Accountability Office's Health Information Technology (HIT) Policy Committee has recently recommended that the Office of the National Coordinator for Health Information Technology include the capture of gender ID data in electronic health records (EHR) as part of Meaningful Use Stage 3. ${ }^{6,7}$ While general recommendations have been published, ${ }^{3}$ recent research demonstrates a wide range of current practices in how this information is captured, and current proposed Systematized Nomenclature in Medicine (SNOMED) codes do not reflect these recommendations. ${ }^{7,} 8$ Barriers to standardization may include lack of specific functionality in a given product, limited understanding of gender identity (gender ID) issues among EHR implementation teams, competing institutional priorities, or a lack of institutional will to address a new and confusing issue. In the rush to develop functionality to meet the upcoming Meaningful Use mandate, it is essential that the issue receive careful consideration to avoid a retrospectively less than ideal outcome and product.

As such, special efforts must be taken to optimize the manner in which gender ID data is collected, stored, and then accessed and displayed in an electronic medical record. While several publications have described what data to collect and how to collect it, limited guidance exists for EHR development teams seeking hard, practical guidance on what actual workflows would look like for end users. This article aims to provide insights into the process based on extensive experience in developing and implementing EHR gender ID functionality at several institutions.

Efforts must begin at registration, where ideally a kiosk or web portal system will allow patients to enter demographic information anonymously, and can increase the likelihood of honest responses. ${ }^{9}$ In the absence of such a self-report system, paper intake forms should be tailored to reflect best practices while minimizing confusion and occupation of valuable space.

Current best practices for the collection of gender ID data (name, gender ID) recommend collection of both gender ID and birth-assigned sex., ${ }^{3,6}$ Transgender people can be identified as those whose gender identity and birth-assigned sex differ. This is known as the "two-step" process and is superior 
NAME: Smith, John*

GENDER: $M *$
DOB: $1 / 1 / 1990$

MR\# 1234567
PCP: Dr. Jones

Insurance: Blue Circle

Figure 1. Gender ID and preferred name differ from birth-assigned and legal data for a transgender patient who has not made any legal document changes. Asterisks serve to denote that preferred name and pronoun which are displayed in the banner differ from those on legal documents. In this case, Gender ID=Male or Transgender male, Birth Sex $=$ Female, Legal Sex $=$ Female, Preferred Name $=\mathbf{J o h n}$, Pronouns $=H e / H i m$.

to a single step that provides choices of "male", "female" and "transgender," as many transgender people will identify as both male or female AND transgender. ${ }^{6}{ }^{10}$

Institutions may have valid concerns regarding collection and use of these data. Safety practices, HIPAA and the Red Flag Rule all require verification of legal identity in settings such as lab, x-ray or procedures. Accurate reporting of legal identity is necessary for appropriate insurance billing. Busy front office staff in settings where most patients are not transgender need to have their overall keystroke load minimized for the majority of their patients. Training needs for clinic providers and staff must be standardized, routinized, and clarified in a way that fits in with many other demands and priorities to insure that current and new hires are fluent in these practices. Here, we attempt to provide an exhaustive description of possible patient scenarios and propose experience-based and evidence-based solutions.

\section{GENDERQUEER, GENDER NONCONFORMING, AND NEUTRAL VERSUS POLAR PRONOUNS}

Some transgender persons may have a gender ID of genderqueer or gender nonconforming; such identities may involve a preference for neutral pronouns as opposed to the traditional polar "he/him" and "she/her". In some cases, the pronouns "they/them" are used and in other cases, the pronouns "zie/hir" are used (pronounced "Zee" and "Here"). Use of the latter may prove difficult to some to master, and as such, the use of "they/them" is a good general recommendation to make for those who identify with neutral pronouns. For example, a provider may call a nurse into the exam room at the end of the visit and say, "They would like to learn more about our online patient portal system. Can you please give them our information pamphlet?"

\section{POTENTIAL SCENARIOS}

1) Transgender person with a gender ID that differs from birth-assigned sex and legal sex (which are the same). Example: gender $\mathrm{ID}=$ male, birth-assigned sex=female, legal sex $=$ female. This would represent a transgender person who has not changed their legal identity documents to reflect their gender ID (Fig. 1).

2) Transgender person with gender ID and legal sex that are the same, and birth-assigned sex that is different. Example: gender $\mathrm{ID}=$ female, birth-assigned sex $=$ male, legal sex $=$ female. This would represent someone who has changed their legal documents to reflect their gender ID (Fig. 2).

3) Transgender person with a gender ID that differs from birth-assigned sex and legal sex (which are also different). Example: gender ID=genderqueer, birthassigned sex $=$ male, legal $s e x=$ female. This would represent a genderqueer-identified person, assigned male at birth, who, when faced with the requirement to choose from either "Male" or "Female" to be listed on legal documents such as a driver license or passport, chooses "Female" (Figs. 3 and 4).

Each of these scenarios has different considerations and requires functionality that permits individualized solutions (Fig. 5).

$\begin{array}{lll}\text { NAME: Smith, Jen } & \text { DOB: } 1 / 1 / 1990 & \text { PCP: Dr. Jones } \\ \text { GENDER: } F & \text { MR\# } 1234567 & \text { Insurance: Blue Circle }\end{array}$

Figure 2. Legal documents have been changed to reflect the legal name and gender ID in a transgender patient with a gender ID that maps to polar (i.e., M/F) pronouns. No asterisks are present; information relevant to their transgender history is contained in the medical history section of the chart. In this case, Gender ID=Female, Birth $S e x=$ Male, Legal Sex=Female, Pronounds=She/Her. 
NAME: Smith, Taryn

GENDER: $\mathrm{N}$ *
DOB: $1 / 1 / 1990$

MR\# 1234567
PCP: Dr. Jones

Insurance: Blue Circle

Figure 3. Legal name has been changed in a genderqueer patient whose gender ID maps to neutral pronouns (regardless of birth sex). Legal sex may or may not have been changed. Asterisk denotes that legal sex differs from gender ID.

\section{GENERAL FUNCTIONALITY RECOMMENDATIONS}

1) Implement three gender fields:

Gender ID

Birth Sex

Legal Sex

2) Implement preferred name field (can use existing nickname or other alias field if such functionality exists).

3) Banner "gender" field would actually indicate preferred pronoun as driven by the Gender ID field, for all patients (details below).

4) Banner "name" field could be driven by legal name for all patients except for those who have an entry in the preferred/ nickname field, the presence of which serves as a Boolean flag to drive the routing of the preferred name to the banner in the place of the legal name. Note this functionality would also have utility for non-transgender patients who have a preferred name or nickname (Fig. 4).

5) Asterisk $(*)$ or other alert icon could be displayed in the banner for any patient whose gender ID $\neq$ legal sex, and/ or any patient whose preferred name $\neq$ legal name. This notifies staff that they must utilize other demographic information not displayed on the banner in order to verify identity, process billing claims, etc....

6) Electronic processes such as billing, printing prescriptions and lab labeling can be driven on the back end by legal sex and legal name fields. Simple patient education materials can explain the need to retain and use legal identifiers in these settings.

7) To minimize registration staff keystrokes, and to minimize confusion for those non-transgender patients who are self-registering via portal or kiosk, demographics input interfaces might only display a field called "Gender," which is actually the "Gender ID" field.
Completion of this gender field will auto-populate the birth sex and legal sex fields, which will not be visible in this view (visible in the demographics section only). $\underline{\mathrm{A}}$ radio button could exist next to the "gender" field, with a label "click here if gender identity differs from birth sex." Upon clicking this button, the birth sex and legal sex fields would appear and could be modified by the patient or by entering staff. Engaging stakeholders and providing culturally grounded initial and ongoing staff training and quality improvement exercises are essential for a successful implementation. The Center of Excellence for Transgender Health at the University of California, San Francisco, has developed an online animated training tool that provides useful guidance on the mechanics of gender ID data collection in a clinical setting (http://transhealth.ucsf.edu/video/story.html).

Note that "banner" includes other locations where name and gender info might be displayed for "quick look" purposes, i.e., a provider's daily schedule, phlebotomy draw schedule, etc....

Medical details of a patient's trans-related history could be kept in the medical/surgical history and problem list area, so providers could see this information when needed.

\section{PRONOUNS}

For non-transgender patients, there is a straightforward and implicit mapping of male or female gender to an " $\mathrm{M}$ " or " $\mathrm{F}$ " gender marker and male (he, him) or female (she, her) pronouns; as such, historically no "pronoun" field has existed in EHR systems, as the user simply looks at the sex/gender field and mentally maps this to the correct pronouns. In the case of transgender patients, this is not so straightforward. Table 1

\begin{tabular}{|lll|}
\hline NAME: Smith, JB * & DOB: $1 / 1 / 1990$ & PCP: Dr. Jones \\
GENDER: $M$ & MR\# 1234567 & Insurance: Blue Circle \\
\hline
\end{tabular}

Figure 4. Preferred name displayed in banner (JB) differs from legal name (John), as indicated by an asterisk. Patient may or may not be transgender; however, gender marker is aligned with legal sex, as indicated by a lack of an asterisk in the gender field. 


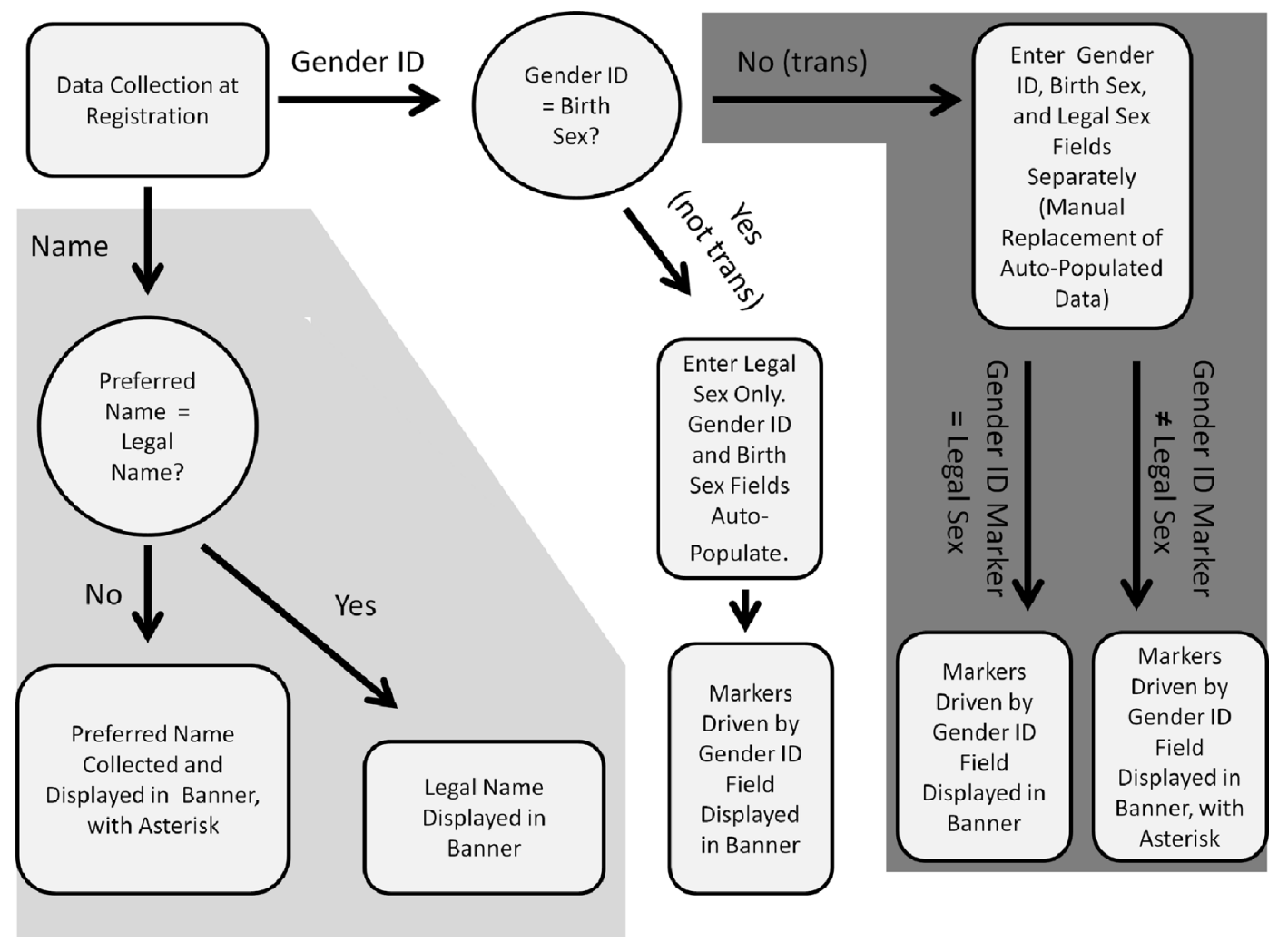

Figure 5. Collection logic flow. Note that the inclusion of a fourth gender ID field for direct entry of preferred pronoun would require additional functionality.

provides a mapping of possible gender ID field values to pronouns and gender markers. Note that this is not a one-toone data relationship, and that more than one gender identity value can map to the same gender marker and pronoun set. Note that some institutions may choose to add a fourth field that allows direct entry of the preferred pronoun, as opposed to the use of this mapping scheme.

Training curricula and materials should provide guidance on how to recognize and use these pronouns, with particular attention to what seems to be the grammatically incorrect use of "they" and "them." Staff inexperienced in transgender care may find these non-binary gender identities and terminologies to be particularly difficult to understand and utilize. Inclusion of an added real-time reminder or popup for patients whose pro-

Table 1. Proposed Mapping of Gender ID, Gender Markers, and Pronouns

\begin{tabular}{lll}
\hline \hline Gender ID & $\begin{array}{l}\text { Gender } \\
\text { Marker }\end{array}$ & Pronoun \\
\hline Male & $\mathrm{M}$ & $\mathrm{He} / \mathrm{Him}$ \\
Female & $\mathrm{F}$ & $\mathrm{She} / \mathrm{Her}$ \\
Transgender male/man, Transman & $\mathrm{M}$ & $\mathrm{He} / \mathrm{Him}$ \\
Transgender female/woman, Transwoman & $\mathrm{F}$ & $\mathrm{She} / \mathrm{Her}$ \\
Genderqueer / Gender nonconforming & $\mathrm{N}$ & They/Them \\
Other Gender Identity & $\mathrm{N}$ & They/Them \\
\hline
\end{tabular}

Figures 1-4: Proposed appearance of banner in various scenarios. Note that while not displayed in the banner, Gender ID, Birth Sex, and Legal Sex would all be stored in the demographics section of the EHR noun preference is "They/Them" within the user interface may help reinforce this behavior among naïve clinic staff and providers. Such a reminder might be designed to popup for a limited number of times per user to reinforce the appropriate use of pronoun and not overburden the user.

The trend of data-to-text functionality within EHR systems that creates "gendered" natural language adds additional challenges in the care of transgender patients. For example, a provider may check a "negative" radio button in the review of systems section under "chest pain"; the data-to-text functionality would then place the phrase "he has not had any chest pain" into the electronic encounter note. Limited data exists evaluating such functionality, and the data that does exist evaluated systems that used natural language to generate chart summaries from expansive data rather than in the context described here, where discrete data points are expanded into natural language expression. In the absence of any data supporting the use of natural-language expansion of point data, particularly with respect to using pronouns (as opposed to more clinical terminology such as "the patient"), we question the expanding role of such functionality in EHRs. ${ }^{11,12}$

\section{CONCLUSION}

Improved, standardized and disseminated best practices for collecting, storing and displaying gender identity data will have a wide range of positive effects on patient satisfaction and 
quality of care, research, funding allocations and policy. Institutional will to undertake the infrastructure and training measures needed to realize these best practices is essential for success. Training efforts should take into consideration that many staff will only serve a handful of transgender patients at most each year, and that staff may vary widely in their ability (or willingness) to master and maintain these skills. A rational approach that engages clinical facility stakeholders is essential. As institutions begin to demand appropriate and insightful EHR functionality, vendors will compete to respond to these demands.

Conflicts of Interest: Madeline B. Deutsch, MD has no conflicts to report.

David Buchholz, MD has no conflicts to report

Corresponding Author: Madeline B. Deutsch, M.D.; Department of Family \& Community Medicine, University of California, 2356 Suffer St, 4th Floor, San Francisco, CA 94115, USA (e-mail: Madeline.Deutsch@ucsf.edu).

\section{REFERENCES}

1. Conron KJ, Scott G, Stowell GS, Landers SJ. Transgender health in Massachusetts: results from a household probability sample of adults. Am J Public Health. 2012;102(1): 118-22.

2. United States Census Bureau. Overview of Race and Hispanic Origin: 2010. Available at https://www.census.gov/2010census/news/releases/ operations/cb11-cn125.html. Accessed October 25, 2014
3. Deutsch MB, Green J, Keatley J, Mayer G, Hastings J, Hall AM, et al. Electronic medical records and the transgender patient: recommendations from the world professional association for transgender health EMR working group. J Am Med Inform Assoc. 2013;20(4):700-3.

4. Bauer GR, Hammond R, Travers R, Kaay M, Hohenadel KM, Boyce M. "I don't think this is theoretical; this is our lives": how erasure impacts health care for transgender people. J Assoc Nurses AIDS Care. 2009;20(5):34861.

5. National Gay And Lesbian Task Force. Injustice at Every Turn: A Report of the National Transgender Discrimination Survey. Available at: http:// endtransdiscrimination.org/PDFs/NTDS_Report.pdf. Accessed October 25, 2014.

6. Cahill S, Makadon H. Sexual orientation and gender identity data collection in clinical settings and in electronic health records: a key to ending LGBT health disparities. LGBT Health. 2014;1(1):34-41.

7. Cahill S, Makadon HJ. Sexual orientation and gender identity data collection update: U.S. Government takes steps to promote sexual orientation and gender identity data collection through Meaningful Use guidelines. LGBT Health. 2014;1(3):157-160.

8. Deutsch MB, Keatley J, Sevelius J, Shade SB. Collection of gender identity data using electronic medical records: survey of current End-user practices. J Assoc Nurses AIDS Care. 2014;25(6):65763

9. Mackenzie SLC, Kurth AE, Spielberg F, Severynen A, Malotte CK, St Lawrence $\mathbf{J}$, et al. Patient and staff perspectives on the use of a computer counseling tool for HIV and sexually transmitted infection risk reduction. J Adolesc Health. 2007;40(6):572-e9.

10. Tate CC, Ledbetter JN, Youssef CP. A Two-question method for assessing gender categories in the social and medical sciences. J Sex Res. 2012;18:110.

11. Scott D, Hallett C, Fettiplace R. Data-to-text summarisation of patient records: using computer-generated summaries to access patient histories. Patient Educ Couns. 2013;92(2):153-9.

12. Hunter J, Freer Y, Gatt A, Reiter E, Sripada S, Sykes C. Automatic generation of natural language nursing shift summaries in neonatal intensive care: BT-nurse. Artif Intell Med. 2012;56(3):157-72. 